\title{
Horizontal gene transfer in parasitic plants
}

Charles C. Davis ${ }^{1}$ and Zhenxiang $\mathrm{Xi}^{1}$

\begin{abstract}
Horizontal gene transfer (HGT) between species has been a major focus of plant evolutionary research during the past decade. Parasitic plants, which establish a direct connection with their hosts, have provided excellent examples of how these transfers are facilitated via the intimacy of this symbiosis. In particular, phylogenetic studies from diverse clades indicate that parasitic plants represent a rich system for studying this phenomenon. Here, HGT has been shown to be astonishingly high in the mitochondrial genome, and appreciable in the nuclear genome. Although explicit tests remain to be performed, some transgenes have been hypothesized to be functional in their recipient species, thus providing a new perspective on the evolution of novelty in parasitic plants.
\end{abstract}

\section{Addresses}

${ }^{1}$ Department of Organismic and Evolutionary Biology, Harvard University, 22 Divinity Avenue, Cambridge, MA 02138, USA

Corresponding author: Davis, Charles C. (cdavis@oeb.harvard.edu)

\section{Introduction}

Parasitism has evolved multiple times across the tree of life. Parasitic plants obtain some or all of their water and nutrients, including carbohydrates and minerals, through a specialized feeding apparatus called the haustorium, which attaches to roots or shoots of their hosts (Figure 1). Parasitic plants exhibit a range of diversity, including species with the ability to photosynthesize (hemiparasites) and those that cannot (holoparasites). Despite the direct 
haustorial connection parasitic plants establish with their hosts, most grow predominantly exterior to their hosts. The exceptions include a small group of endophytic holoparasites, which emerge only during sexual reproduction (Figure 1). These species otherwise grow embedded in their hosts and have no discernable roots, shoots, or leaves, persisting largely as a mycelium-like body consisting of a greatly reduced strand of cells [1•].

Owing to their extreme vegetative reduction and modified reproductive morphology, the phylogenetic placement of parasitic plants among their freeliving relatives was long a mystery. This is especially true for the endophytic holoparasites, most of which have been historically grouped together to include species variously placed today in Apodanthaceae, Cytinaceae, Mitrastemonaceae, and Rafflesiaceae. Recent phylogenetic investigations, however, have greatly challenged this traditional view of classification by demonstrating that these families are not closely related. Instead, the most comprehensive analyses indicate that parasitism in angiosperms has evolved at least 11 times from free-living ancestors [2].

These insights have greatly stimulated research relating to genome evolution in parasitic plants $[3 \bullet \bullet, 4]$, and the investigation of the adaptations that have enabled the origin of parasitism $[5 \bullet \bullet, 6,7]$. One of the most exciting discoveries to emerge from this body of research is the finding that parasitic plants and their hosts undergo horizontal gene transfer (HGT)-the exchange of genetic materials between distantly related, non-mating organisms. More generally, the hypothesis of HGT in autotrophic plants was invoked in two landmark studies [8,9], in which gene phylogenies were identified as strongly incongruent with well-established species relationships based on various lines of molecular and morphological evidence. In this regard, phylogenies that are incongruent with accepted species relationships have been deemed the 'gold standard' for deducing HGT [10] (Figure 2). Since that time, HGT has been identified in a variety of autotrophic

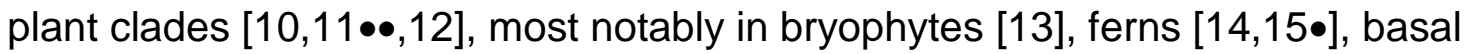
angiosperms [16,17••], and grasses [18].

\section{Parasitic plants are exemplary systems for studying HGT}

Following the two initial discoveries of HGT in autotrophic plants, investigations in parasitic plants have provided important insights into HGT [14,19-21]. Several studies ranging from single to hundreds of genes demonstrate that the parasitic mode of life may enable HGT in plants, which is thought to be facilitated by the 
intimate physical association between the parasite and its host [19]. This intimacy makes parasitic plants a potential model system for HGT related research. Here, the donor and recipient lineages are often well characterized, making it much easier to discern HGT. In contrast, explanations of HGT in autotrophic plants tend to be less clear from a mechanistic perspective. Up until now, both host-toparasite [e.g., 19] and parasite-to-host gene transfers [e.g., 14,20] have been identified in parasitic plants (Table 1). In addition, the possibility that parasitic plants act as bridges for gene flow between diverse autotrophic plants has been invoked [16]. Because most HGTs reported to date involve host-to-parasite transfers, we focus our review on transgenes identified in parasitic plants, and draw upon broader HGT studies in autotrophic plants when they are especially relevant. These include: the phylogenetic breadth and magnitude of HGT in parasitic plants, the genomic compartmentalization of transgenes including potential mechanisms for how these events occur, the functionality of transgenes in their recipient species, and lastly, insights into past parasite-host associations from HGT.

\section{Parasitic plants show high rates of HGT}

Evidence of HGT has been identified in 10 of the 11 parasitic lineages to date (Table 1) and overwhelmingly involve mitochondrial DNA, which is consistent with most studies from autotrophic plants $[17 \bullet \bullet, 22]$. This is likely due to several unique properties of mitochondria, including their ability to actively uptake DNA [23], and to undergo frequent fusion and fission [24]. Additionally, plant mitochondrial genomes possess massive intergenic regions [25], allowing for foreign genes to be inserted without disturbing expressed native genes. In the first broad genomic study to comprehensively assess the magnitude of HGT involving host-parasite mitochondrial genomes, $\mathrm{Xi}$ et al. [3••] identified that up to $41 \%$ of mitochondrial genes in Rafflesiaceae demonstrate evidence of HGT. Here, four mitochondrial genes maintain both horizontally and vertically transferred homologs (duplicative HGT, sensu [11••]), and seven include only transgenic sequences (Figure 2). Moreover, at least some of these transgenes maintain synteny with their hosts, suggesting that native genes were likely displaced via homologous recombination (replacement HGT, sensu [11••]). Subsequent efforts to assemble a mitochondrial genome in Rafflesiaceae support these findings [26•], and further point towards the complete loss of the plastid genome in these endophytic holoparasites. While these results of HGT are lower than the nearly six genome equivalents of DNA content transferred into the autotrophic species Amborella trichopoda [17••], they clearly indicate that a 
parasitic mode of life can significantly reshape the content of mitochondrial genomes via HGT.

In contrast, HGT involving plastid DNA is rare [11••,27]. This is likely due to the fact that plastids do not have DNA uptake mechanisms, and plastid genomes have few intergenic regions. A recent HGT was invoked for the plastid gene rps2 in Phelipanche (Orobanchaceae; in this case HGT involved parasite-to-parasite transfer). Here, the researchers were agnostic on the genomic localization of rps2. One possibility is that the transgene moved directly from the host's plastid genome to the parasite's mitochondrial genome. A second is that this transgene resulted from intergenomic transfer, first involving plastid-to-mitochondrion transfer within the donor lineage, followed by mitochondrion-to-mitochondrion host-to-parasite transfer. With regard to the second possibility, a clearer example was demonstrated in Rafflesiaceae, where Sapria himalayana [3••] and Rafflesia lagascae [26•] acquired an astonishing 14 and 15 plastid genes from their grapevine hosts (Tetrastigma, Vitaceae), respectively. Based on the availability of a previously assembled mitochondrial genome of the host lineage [28], it was confidently determined that these transgenes were likely resident in the host's mitochondrial genome at the time of transfer. These results collectively establish that most transgenes to date have been acquired by the mitochondrial genome

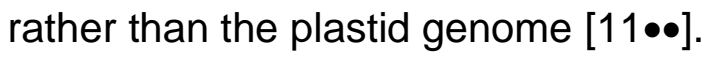

Although a larger picture of rampant HGT involving mitochondrial DNA in parasitic plants is emerging, our knowledge of HGT involving nuclear DNA is still in its infancy. This is partly due to the paucity of deeply sequenced genomes available to address this topic in a broad, comparative framework. Yoshida et al. [29] used genome scanning approaches to demonstrate that the gene ShContig9483 in the purple witchweed, Striga hermonthica (Orobanchaceae), was acquired from a member of the grass family (Poaceae). Similar host-toparasite transfers have subsequently been identified in Cuscuta australis and Orobanche aegyptiaca (Convolvulaceae and Orobanchaceae, respectively; involving the strictosidine synthase-like gene) [30] and Phelipanche aegyptiaca (involving the albumin 1 gene) [31•]. More focused efforts investigating hundreds of genes indicate that host-to-parasite transfer involving nuclear genes may be prevalent. For example, several dozen genes were identified via phylotranscriptomics as likely candidates for nucleus-to-nucleus transfers between Rafflesiaceae and their hosts [4]. These authors additionally sought to establish background rates of non-HGT causes of phylogenetic incongruence 
(e.g., gene duplication, gene loss, and incomplete lineage sorting). This is likely to become especially relevant as more genomic data become available. Promising new analytical approaches have been developed to identify HGT in the face of these confounding effects using parsimony [32,33] or Bayesian methods $[34,35 \bullet]$.

In summary, these results suggest that while the relative magnitude of HGT involving the nuclear genome in parasitic plants is appreciable, transfers involving the mitochondrial genome are likely to be potentially much more frequent.

\section{Mechanisms and functionality of transgenes in parasitic plants}

One outstanding question involves the uptake mechanisms of foreign DNA. While vectors remain unclear, fungi, bacteria, and viruses have been invoked $[9,10]$. More recently, transposable elements have also been implicated as a vector for HGT in autotrophic plants [36•]. While many nutrients and macromolecules, including mRNAs, are trafficked between host and parasite $[5 \bullet \bullet, 37,38]$, the evidence points primarily to direct uptake of DNA, rather than mRNA. This is demonstrated in various studies of parasitic plants [3••,4,22,30,31•], as well as in autotrophic ones. In Rafflesiaceae, evidence for direct DNA uptake is supported by the presence of introns in transgenes and the inclusion of cytosine-to-uracil RNA editing sites in mitochondrial transgenes. One exception is in Striga where the putative transgene mentioned above possesses no intron and has consecutive adenine nucleotides at the 3' end, suggesting that this transgene was RNA-mediated and then incorporated into the genome via reverse transcription [29]. Given the unique properties of the mitochondrial genome and recent observation of exchange of whole plastid genomes in plant grafting experiments $[39,40]$, it appears that the intimate association between parasites and their hosts greatly facilitates HGT via the direct incorporation of large fragments of DNA, rather than shorter mRNAs. Finally, as broader comparative data becomes available on the sizes of plasmodesmatal connections between hosts and parasites, we may gain insight into which symbioses are likely to confer more or less HGT.

A second outstanding question relates to the functionality of transgenes in parasitic plants. Although the magnitude of HGT involving nuclear genes is lower, on average, than that of the mitochondrial genome, nuclear transgenes may nevertheless confer key evolutionary advantages in their recipient lineages. Most of the acquired DNAs identified in mitochondrial genomes are introns or 
pseudogenes. Whether these pseudogenes are inherited as such at the time of transfer, or whether gene death takes place subsequent to their transfer via deterioration of the foreign DNA remains unclear [12]. However, several recently identified nuclear transgenes maintain reading frame, and in some cases have been shown to be actively transcribed. The Rafflesia cantleyi transcriptome, for example, exhibits dozens of expressed transgenes that represent a wide range of cellular functions as determined by gene annotation data, including roles related to respiration, metabolism, mitochondrial translation, and protein turnover [4]. Although the extent to which these transgenes are functional awaits further exploration, these results raise the possibility that transcripts acquired via HGT may express host-like genes to better manipulate their host. Similarly, a transgene closely related to the albumin 1 gene was discovered in Phelipanche aegyptiaca [31 •]. albumin 1 genes encode seed storage proteins and insect toxins, and appear to have been acquired via HGT in the ancestor of Phelipanche from one of its legume hosts. In this case, the transfer appears to have given rise to a family of duplicated, differentially expressed genes in their recipient lineage. This suggests the intriguing possibility that differential function of these copies may have arisen in the parasites subsequent to their transfer. In these cases, the functional relevance of transgenes remains unclear, but parasitic plants need to avoid host defenses as well as resist herbivores, bacteria, fungi, and abiotic environmental stresses. Follow-up studies may reveal that these transgenes provide an evolutionary advantage in this regard.

Finally, an additional discovery to emerge is that codon usage patterns of native genes in some parasites demonstrate a very strong genome wide bias towards coding properties more like those of their hosts rather than their closest relatives [4]. This apparent form of molecular convergence may help to explain why HGT is so high in some parasitic lineages: the similarity in their genetic machinery relative to their hosts may greatly facilitate gene uptake. Why this convergence exists remains an open topic for investigation, and should be investigated in other lineages.

\section{Detecting former host associations with HGT}

Perhaps one of the most exciting possibilities offered by HGT in parasitic systems is their ability to provide insights into current and past host associations. In a recent broad survey of Rafflesiaceae mitochondrial genomes [3••], gene transfers fell within two distinct categories-transfers that were ancient, perhaps dating back to the late Cretaceous, and those that were more recent. For the ancient transfers, transgenes were broadly shared and maintained synteny 
across related parasitic plants. This combination of factors suggests that they were likely the result of a single, ancient host-to-parasite transfer event. In other cases, however, transgenes from these parasitic plants were broadly dispersed among more recently derived host lineages, suggesting recent transfers. Interestingly, in the case of the ancient transfers, the transgenes did not group near to their current host lineages, suggesting that HGT involved former host associations, subsequently followed by a host shift. This is further supported by molecular divergence estimates, which demonstrate that the origin of the parasitic plants greatly predates that of their extant hosts [3••]. In contrast, the more recent transfers tended to group with their current hosts. In these regards, HGT can serve as the 'ghost of transfers past', and will aid us in identifying former host associations. As more and better comparative genome data become available we will be able to time these HGT events with greater precision to better identify former host associations. In addition, by identifying these former associations we can begin to clarify fundamental host requirements of these parasitic plants.

\section{Conclusions and future directions}

Studies to date indicate that parasitic plants represent an active area of HGT. These symbioses are ideal for studying this phenomenon owing to the intimacy of their symbiotic interactions, and our ability to more confidently invoke HGT when phylogenies are incongruent with accepted species relationships. In general, HGT in parasitic plants is reflective of what we see in autotrophic plants [10,11••]: gene transfers involving mitochondrial genomes are high, and appreciable in nuclear genomes. The functionality of these transgenes is unknown, but seem likely in at least some instances. Along these lines, establishing functionality of transgenes in their recipient genomes will be a key goal going forward. Despite lower overall rates of HGT in the nuclear genome, these events could have a major influence on the evolution of novelty in their recipient lineages. The difficulty in elucidating function is not trivial, however, owing to their two-parted symbiosis and the challenges of growing these plants in controlled environments. In this regard, some taxa, such as Cuscuta, are likely to be more suitable for this purpose [41]. These species possess a simple anatomical structure, seed germination requiring only water, and a wide range of host tolerances. In addition to these more phylogenetically focused experiments, HGT remains largely unexplored for numerous parasitic clades at the genomic level. Future explorations should generate genomic data for these neglected clades to further discern the magnitude of HGT, especially by sequencing regions outside of transgenes to identify putative vectors from genomic 
signatures. These exercises in genomic surveillance should prove useful for understanding differential rates of HGT among genomes, common patterns of genes that are transferred (and lost in recipient genomes following transfer), and the prevalence of HGT among different modes of parasitism.

\section{Acknowledgements}

CCD and ZX were supported by National Science Foundation (NSF) Assembling the Tree of Life grant DEB-0622764 and NSF DEB-1120243 (to CCD). Members of the Davis laboratory provided helpful feedback on an early draft of this paper.

\section{References and recommended reading}

Papers of particular interest, published within the period of review, have been highlighted as:

- of special interest

$\bullet$ of outstanding interest

1. Nikolov LA, Tomlinson PB, Manickam S, Endress PK, Kramer EM, Davis CC: Holoparasitic Rafflesiaceae possess the most reduced endophytes and yet give rise to the world's largest flowers. Ann Bot 2014, 114:233-242.

- This study characterizes the anatomy and morphology of the greatly reduced endophyte of the parasitic plant clade, Rafflesiaceae, which also produce the world's largest flowers. It provides a good introduction to holoparasites more generally.

2. Barkman TJ, McNeal JR, Lim SH, Coat G, Croom HB, Young ND, dePamphilis CW: Mitochondrial DNA suggests at least 11 origins of parasitism in angiosperms and reveals genomic chimerism in parasitic plants. BMC Evol Biol 2007, 7:248.

3. Xi Z, Wang Y, Bradley RK, Sugumaran M, Marx CJ, Rest JS, Davis CC: Massive mitochondrial gene transfer in a parasitic flowering plant clade. PLoS Genet 2013, 9:e1003265.

-• This is the first study to comprehensively assess the magnitude of HGT in plants involving a genome (mitochondrion) and a species interaction (parasitism) where it is thought to be rampant. Up to $41 \%$ of mitochrondrial genes in these species are determined to have undergone HGT. And evidence of homologous recombination of transgenes with portions of native genes combined to form genes of chimeric origin. 
4. Xi Z, Bradley RK, Wurdack KJ, Wong K, Sugumaran M, Bomblies K, Rest JS, Davis CC: Horizontal transfer of expressed genes in a parasitic flowering plant. BMC Genomics 2012, 13:227.

5. Kim G, LeBlanc ML, Wafula EK, dePamphilis CW, Westwood JH: Genomicscale exchange of mRNA between a parasitic plant and its hosts. Science 2014, 345:808-811.

-• This study identifies the large-scale bidirectional exchange of mRNA between host and parasite, and suggests this as a new 'language' between these interacting species.

6. Westwood JH, Yoder JI, Timko MP, dePamphilis CW: The evolution of parasitism in plants. Trends Plant Sci 2010, 15:227-235.

7. Ichihashi Y, Mutuku JM, Yoshida S, Shirasu K: Transcriptomics exposes the uniqueness of parasitic plants. Brief Funct Genomics 2015, 14:10.1093/bfgp/elv001.

8. Won $\mathrm{H}$, Renner SS: Horizontal gene transfer from flowering plants to Gnetum. Proc Natl Acad Sci USA 2003, 100:10824-10829.

9. Bergthorsson U, Adams KL, Thomason B, Palmer JD: Widespread horizontal transfer of mitochondrial genes in flowering plants. Nature 2003, 424:197-201.

10. Keeling PJ, Palmer JD: Horizontal gene transfer in eukaryotic evolution. Nat Rev Genet 2008, 9:605-618.

11. Sanchez-Puerta MV: Involvement of plastid, mitochondrial and nuclear genomes in plant-to-plant horizontal gene transfer. Acta Soc Bot Pol 2014, 83:317-323.

-• This a recent review of HGT in plants, not specifically focused on parasitic plants.

12. Bock R: The give-and-take of DNA: horizontal gene transfer in plants. Trends Plant Sci 2010, 15:11-22.

13. Yue J, Hu X, Sun H, Yang Y, Huang J: Widespread impact of horizontal gene transfer on plant colonization of land. Nat Commun 2012, 3:1152.

14. Davis CC, Anderson WR, Wurdack KJ: Gene transfer from a parasitic flowering plant to a fern. Proc $R$ Soc $B$ 2005, 272:2237-2242.

15. Li F-W, Villarreal JC, Kelly S, Rothfels CJ, Melkonian M, Frangedakis E, Ruhsam M, Sigel EM, Der JP, Pittermann J, et al.: Horizontal transfer of an adaptive chimeric photoreceptor from bryophytes to ferns. Proc Natl Acad Sci USA 2014, 111:6672-6677.

- This study identifies a photoreceptor gene transferred from bryophytes (hornwort) to a fern. This gene may play an adaptive role in the recipient fern lineage, but this remains untested. 
16. Bergthorsson U, Richardson AO, Young GJ, Goertzen LR, Palmer JD: Massive horizontal transfer of mitochondrial genes from diverse land plant donors to the basal angiosperm Amborella. Proc Natl Acad Sci USA 2004, 101:17747-17752.

17. Rice DW, Alverson AJ, Richardson AO, Young GJ, Sanchez-Puerta MV, Munzinger Jrm, Barry K, Boore JL, Zhang Y, dePamphilis CW, et al.: Horizontal transfer of entire genomes via mitochondrial fusion in the angiosperm Amborella. Science 2013, 342:1468-1473.

-• This paper reports the nearly 4-megabase mitochondrial genome of Amborella, which acquired six genome equivalents of foreign DNA via HGT from donors as diverse as algae and mosses. How this occurred is unclear but may have been facilitated by the close interaction between Amborella and its diverse leaf epiphyte community.

18. Christin P-A, Edwards EJ, Besnard G, Boxall SF, Gregory R, Kellogg EA, Hartwell J, Osborne CP: Adaptive evolution of C4 photosynthesis through recurrent lateral gene transfer. Curr Biol 2012, 22:445-449.

19. Davis CC, Wurdack KJ: Host-to-parasite gene transfer in flowering plants: phylogenetic evidence from Malpighiales. Science 2004, 305:676678.

20. Mower JP, Stefanovic S, Young GJ, Palmer JD: Gene transfer from parasitic to host plants. Nature 2004, 432:165-166.

21. Nickrent DL, Blarer A, Qiu Y-L, Vidal-Russell R, Anderson FE: Phylogenetic inference in Rafflesiales: the influence of rate heterogeneity and horizontal gene transfer. BMC Evol Biol 2004, 4:40.

22. Mower JP, Stefanovic S, Hao W, Gummow JS, Jain K, Ahmed D, Palmer JD: Horizontal acquisition of multiple mitochondrial genes from a parasitic plant followed by gene conversion with host mitochondrial genes. $B M C$ Biol 2010, 8:150.

23. Koulintchenko M, Konstantinov Y, Dietrich A: Plant mitochondria actively import DNA via the permeability transition pore complex. EMBO J 2003 , 22:1245-1254.

24. Arimura S, Yamamoto J, Aida GP, Nakazono M, Tsutsumi N: Frequent fusion and fission of plant mitochondria with unequal nucleoid distribution. Proc Natl Acad Sci USA 2004, 101:7805-7808.

25. Kitazaki K, Kubo T: Cost of having the largest mitochondrial genome: evolutionary mechanism of plant mitochondrial genome. J Bot 2010, 620137.

26. Molina J, Hazzouri KM, Nickrent D, Geisler M, Meyer RS, Pentony MM, Flowers JM, Pelser P, Barcelona J, Inovejas SA, et al.: Possible loss of the 
chloroplast genome in the parasitic flowering plant Rafflesia lagascae (Rafflesiaceae). Mol Biol and Evol 2014, 31:793-803.

- This study characterizes the likely loss of the plastid genome in the holoparasitic plant clade, Rafflesiaceae.

27. Rice DW, Palmer JD: An exceptional horizontal gene transfer in plastids: gene replacement by a distant bacterial paralog and evidence that haptophyte and cryptophyte plastids are sisters. BMC Biol 2006, 4:31.

28. Goremykin VV, Salamini F, Velasco R, Viola R: Mitochondrial DNA of Vitis vinifera and the issue of rampant horizontal gene transfer. Mol Biol and Evol 2009, 26:99-110.

29. Yoshida S, Maruyama S, Nozaki H, Shirasu K: Horizontal gene transfer by the parasitic plant Striga hermonthica. Science 2010, 328:1128.

30. Zhang D, Qi J, Yue J, Huang J, Sun T, Li S, Wen J-F, Hettenhausen C, Wu J, Wang L, et al:: Root parasitic plant Orobanche aegyptiaca and shoot parasitic plant Cuscuta australis obtained Brassicaceae-specific strictosidine synthase-like genes by horizontal gene transfer. BMC Plant Biol 2014, 14:19.

31. Zhang $Y$, Fernandez-Aparicio M, Wafula E, Das M, Jiao $Y$, Wickett $N$, Honaas L, Ralph P, Wojciechowski M, Timko M, et al.: Evolution of a horizontally acquired legume gene, albumin 1, in the parasitic plant Phelipanche aegyptiaca and related species. BMC Evol Biol 2013, 13:48.

-• This study traces a nuclear transgene from the parasitic plant Phelipanche to their distant legume donor. The authors identify the evolutionary fate of this transgene in its recipient lineage, which goes on to evolve and possibly specialize to achieve new function within the parasite.

32. Chen K, Durand D, Farach-Colton M: NOTUNG: a program for dating gene duplications and optimizing gene family trees. J Comput Biol 2000, 7:429447.

33. Stolzer M, Lai H, Xu M, Sathaye D, Vernot B, Durand D: Inferring duplications, losses, transfers and incomplete lineage sorting with nonbinary species trees. Bioinformatics 2012, 28:i409-i415.

34. Szöllösi GJ, Boussau B, Abby SS, Tannier E, Daubin V: Phylogenetic modeling of lateral gene transfer reconstructs the pattern and relative timing of speciations. Proc Natl Acad Sci USA 2012, 109:17513-17518.

35. Sjöstrand J, Tofigh A, Daubin V, Arvestad L, Sennblad B, Lagergren J: A Bayesian method for analyzing lateral gene transfer. Syst Biol 2014, 63:409-420.

- This is a new Bayesian method for assessing gene duplication and gene loss versus horizontal gene transfer within a phylogenomic data set. 
36. El Baidouri M, Carpentier M-C, Cooke R, Gao D, Lasserre E, Llauro C, Mirouze M, Picault N, Jackson SA, Panaud O: Widespread and frequent horizontal transfers of transposable elements in plants. Genome Res 2014, 24:831-838.

- This study uses analyses of whole genome sequences to establish that HGT involving transposable elements may be common in plants.

37. Westwood JH, Roney JK, Khatibi PA, Stromberg VK: RNA translocation between parasitic plants and their hosts. Pest Manag Sci 2009, 65:533539.

38. Roney JK, Khatibi PA, Westwood JH: Cross-species translocation of mRNA from host plants into the parasitic plant dodder. Plant Physiol 2007, 143:1037-1043.

39. Stegemann S, Keuthe M, Greiner S, Bock R: Horizontal transfer of chloroplast genomes between plant species. Proc Natl Acad Sci USA 2012, 109:2434-2438.

40. Stegemann S, Bock R: Exchange of genetic material between cells in plant tissue grafts. Science 2009, 324:649-651.

41. Furuhashi T, Furuhashi K, Weckwerth W: The parasitic mechanism of the holostemparasitic plant Cuscuta. J Plant Interact 2011, 6:207-219.

42. Park JM, Manen JF, Schneeweiss GM: Horizontal gene transfer of a plastid gene in the non-photosynthetic flowering plants Orobanche and Phelipanche (Orobanchaceae). Mol Phylogenet Evol 2007, 43:974-985.

43. Li X, Zhang TC, Qiao Q, Ren Z, Zhao J, Yonezawa T, Hasegawa M, Crabbe MJ, Li J, Zhong Y: Complete chloroplast genome sequence of holoparasite Cistanche deserticola (Orobanchaceae) reveals gene loss and horizontal gene transfer from its host Haloxylon ammodendron (Chenopodiaceae). PLOS ONE 2013, 8:e58747.

Figure 1. Parasitic plant diversity. 1) Holoparasitic Cuscuta europaea (Convolvulaceae); 2) Cuscuta campestris (Convolvulaceae) penetrating host tissue via a haustorium (highlighted with dotted yellow lines); 3) Holoparasitic Rafflesia arnoldii (Rafflesiaceae), which produces the world's largest flowers; 4) Holoparasitic Rhizanthes lowii (Rafflesiaceae) showing its very reduced endophyte (marked with dotted yellow lines) in the phloem of the host-the only remnants of a vegetative body; 5) Hemiparasitic Taxillus delavayi (Loranthaceae, Santalales); 6) Hemiparasitic Krameria argenteal (Krameriaceae); 7) Holoparasitic Pilostyles hamiltonii (Apodanthaceae); 8) Holoparasitic Mitrastema yamamotoi (Mitrastemonaceae); 9) Holoparasitic Pholisma sonorae 
(Lennoaceae); and 10) Holoparasitic Cistanche phelyphaea (Orobanchaceae). Images copyright Dave, M. Costea, J. Holden, L. Nikolov, J. Lundberg, J. Medeiros, K. Thiele, C. Tada, J. Bartel, and P. Precey, respectively.

Figure 2. Using phylogeny to infer horizontal gene transfer (HGT). a) Inferred gene tree on the left depicts transgene in parasite (placed with host clade), coexisting with its vertically inherited homolog (i.e., duplicative HGT). Outer species tree (in grey) on the right with inner gene tree demonstrating host-toparasite HGT. b) Inferred gene tree on the left depicts transgene in parasite (placed with host clade). Outer species tree on the right with inner gene tree demonstrating host-to-parasite HGT, followed by loss of vertically inherited homolog (red ' $x$ '; i.e., replacement HGT).

Table 1. Summary of horizontal gene transfer (HGT) involving parasitic plants.

\begin{tabular}{lll}
\hline Clade & Reference & Transgenes \\
\hline Apodanthaceae & {$[21]$} & 1 mitochondrial gene \\
& {$[2]$} & 2 mitochondrial genes \\
$\begin{array}{l}\text { Cassytha } \\
\text { (Lauraceae) }\end{array}$ & {$[2]$} & 1 mitochondrial gene \\
$\begin{array}{l}\text { Cuscuta } \\
\text { (Convolvulaceae) }\end{array}$ & {$[20]$} & 1 mitochondrial gene \\
& {$[22]$} & 3 mitochondrial genes \\
Cynomoriaceae & {$[2]$} & 2 mitochondrial genes \\
Hydnoraceae & {$[2]$} & 1 mitochondrial gene \\
Lennooideae & {$[2]$} & 1 mitochondrial gene \\
(Boraginaceae) & & \\
Mitrastemonaceae & {$[21]$} & 1 mitochondrial gene \\
& {$[2]$} & 2 mitochondrial genes \\
Orobanchaceae & {$[20]$} & 1 mitochondrial gene \\
& {$[42]$} & 1 plastid gene \\
& {$[29]$} & 1 nuclear gene \\
& {$[43]$} & 1 plastid gene \\
& {$[31 \bullet$} & 1 nuclear gene \\
& {$[30]$} & 1 nuclear gene
\end{tabular}




\begin{tabular}{lll} 
Rafflesiaceae & {$[19]$} & 1 mitochondrial gene \\
& {$[21]$} & 1 mitochondrial gene \\
& {$[2]$} & 1 mitochondrial gene \\
& {$[4]$} & 47 nuclear and 2 mitochondrial genes \\
& {$[3 \bullet \bullet$} & 11 mitochondrial and 14 plastid genes \\
Santalales & {$[26 \bullet]$} & 15 plastid genes \\
& {$[14]$} & 2 mitochondrial genes \\
& {$[2]$} & 1 mitochondrial gene \\
\hline
\end{tabular}




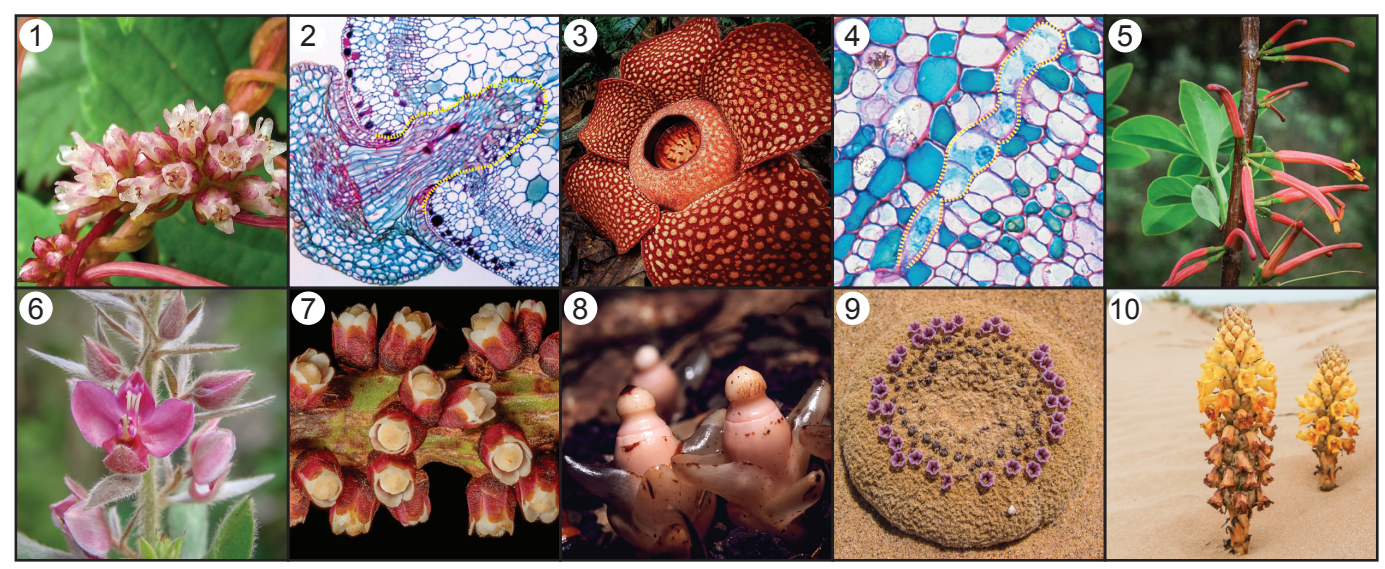

Page 16 of 17 

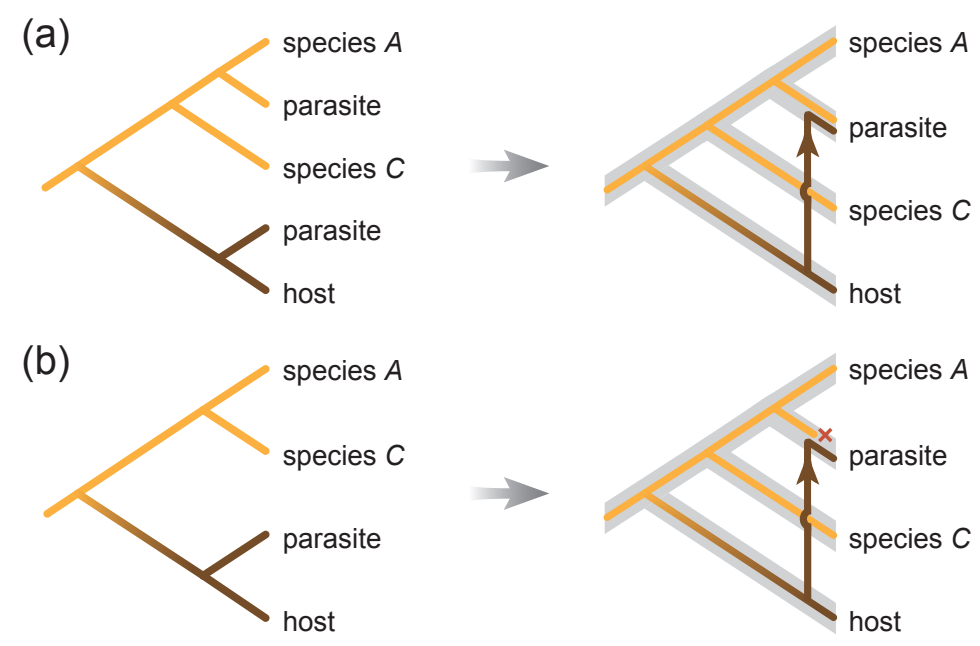